\title{
High-pressure $\mathrm{CO}_{2}$ for lipid-mediated cocrystallization of theophylline and nicotinamide
}

\author{
$<\llbracket$ Tatsumi, Y. Hao, Y. Shimoyama* \\ Department of Chemical Science and Engineering, Tokyo Institute of Technology, Japan \\ S1-33 2-12-1 Ookayama, Meguro-ku, Tokyo 152-8550 Japan \\ *yshimo@chemeng.titech.ac.jp
}

In order to improve solubility and dissolution rate of pharmaceutical products in human body, the cocrystal formation has been focused on. The cocrystal allows to form the new crystal structure composed of active pharmaceutical ingredients (APIs) and additives called as coformer (CF). Most conventional methods require a treatment in organic solvent to form cocrystal, so they include disadvantages on the safety due to remaining the organic solvent [1]. A hot melt extrusion, which is the cocrystal formation process using liquefied polymer, may include a thermal decomposition of APIs by heating process at high temperature to melt polymer [2]. In this study, we focus on melting point depression of lipids by high pressure $\mathrm{CO} 2$. In this research, we propose a cocrystallization process with liquefied lipid under high pressure $\mathrm{CO}_{2}$ (Fig. 1).

In this study, we use theophylline (TPL) as API, nicotinamide (NA) as CF. In addition, stearic acid (SA), oleic acid (OA) and linoleic acid (LA) whose unsaturation is each 0,1 and 2 are used as lipids. The carbon numbers of all the lipids are. For comparison, we conducted experiments without lipids and experiments using hydrocarbon with the same carbon number, octadecane (OD) and 1-octadecene (1OD). We put TPL $(0.1 \mathrm{mmol})$, NA $(0.1 \mathrm{mmol})$ and lipid $(0.04 \mathrm{~g})$ in high-pressure vessel under $16.0 \mathrm{MPa}$ at $50^{\circ} \mathrm{C}\left(60^{\circ} \mathrm{C}\right.$ only SA) for 2 h. Moreover, the experiments were conducted in which TPL $(1 \mathrm{mmol})$, NA $(1 \mathrm{mmol})$ and lipid $(0.4 \mathrm{~g})$ were being stirred at $300 \mathrm{rpm}$ for $2 \mathrm{~h}$ under same the temperature and pressure conditions.

As the X-ray diffraction results, cocrystal formations are improved by liquified lipid compared with those formed in hydrocarbon or without lipids. Moreover, the conversion rate of cocrystal formation is evaluated from the peak intensity ratio of XRD between TPL and cocrystal by RIR method, which is a way to evaluate cocrystal's purity using that the mass ratio in the mixture is proportional to the characteristic peak intensity ratio [3]. As a result, it is found out that cocrystal formation is improved as the degree of lipid unsaturation increased (Fig. 2). Similarly, in the cases with stirring, Fig. 2 shows that lipids with high unsaturation promotes cocrystallization than hydrocarbons. These results could be due to the facilitation of interactions between TPL or NA surfaces and lipid surfaces by carboxyl groups in lipids, which may cause the activation of each interface during cocrysallization. In addition, to explain the facilitation of interactions by lipids, we calculate the molecular interaction energy among TPL, NA and lipid by Conductor-like Screening Model (COSMO) [4]. As a result, the comparison between calculated and experimental results suggests that cocrystal formation is promoted by increasing molecular interactions. Therefore, we conclude that lipids with big interaction energy with API and CF help to form cocrystal under high pressure $\mathrm{CO}_{2}$.

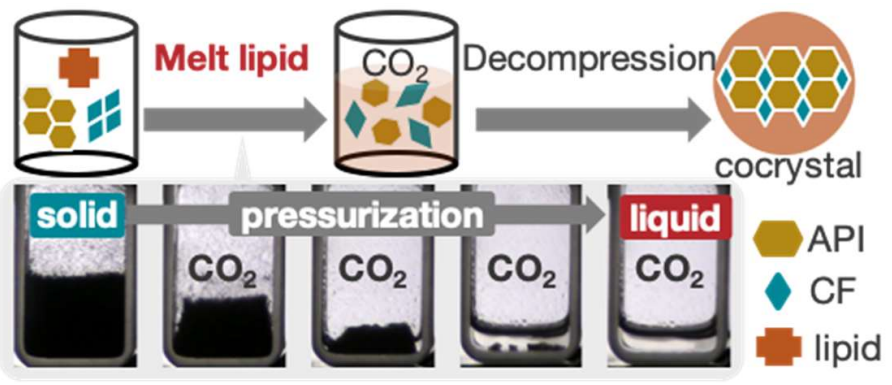

Figure 1. Lipid-mediated cocrystal formation by high pressure $\mathrm{CO}_{2}$

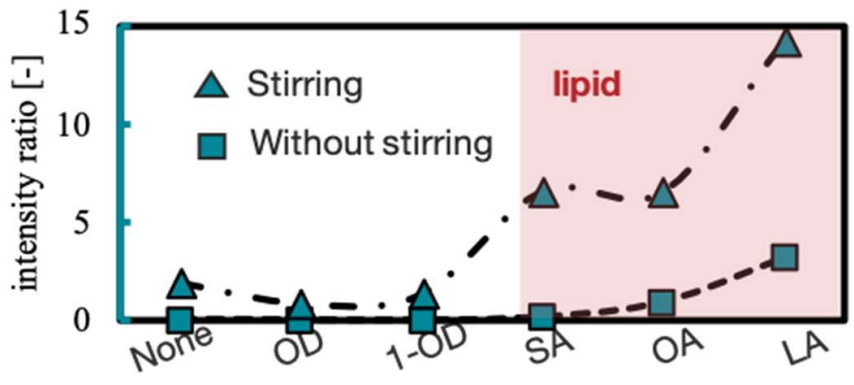

Figure 2. Cocrystal conversion rate by lipid

[1] Ping L., Yueying C., Lin W., Robert M. W, Jr., Kaichao Y., Hailu Z. \& Zongwu D. (2014). Royal Society of Chemistry, 16, $3141-3147$.

[2] Priyanka S., Mashan A., Nagireddy D., Sandeep S., Suresh B., Feng Z., Eman A. \& Michael A. R. (2021). J. Drug Deliv. Sci. Technol. 61, 102128.

[3] Hideo T. (2018). J. Appl. Cryst. 51, 446-455.

[4] Shiang T. L. \& Stanley I. S. (2002). Ind. Eng. Chem. Res. 41, 899-913.

Keywords: high-pressure $\mathrm{CO}_{2}$; lipid-mediated; cocrystal

This research was supported by Japan Society for the Promotion of Science, Grant-in-Aid for Challenging Research (Pioneering) $20 K 21105$.

Acta Cryst. (2021), A77, C1256 